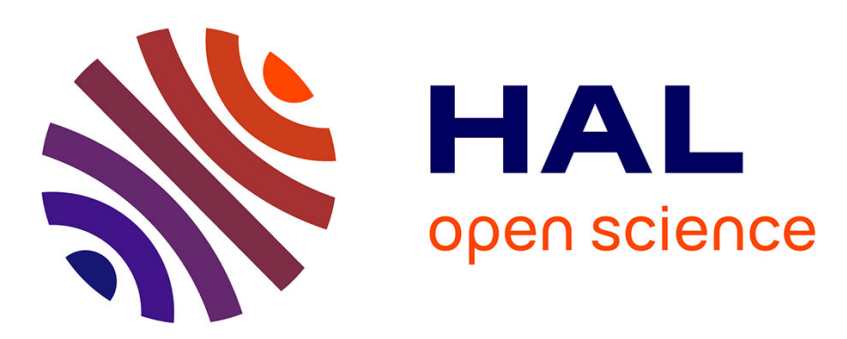

\title{
A new cloud-based hp finite element method
}

\author{
J.T. Oden, C.A.M. Duarte, O.C. Zienkiewicz
}

\section{To cite this version:}

J.T. Oden, C.A.M. Duarte, O.C. Zienkiewicz. A new cloud-based hp finite element method. Computer Methods in Applied Mechanics and Engineering, 1998, 153 (1-2), pp.117 - 126. 10.1016/S00457825(97)00039-X . hal-01722149

\section{HAL Id: hal-01722149 \\ https://hal.science/hal-01722149}

Submitted on 3 Mar 2018

HAL is a multi-disciplinary open access archive for the deposit and dissemination of scientific research documents, whether they are published or not. The documents may come from teaching and research institutions in France or abroad, or from public or private research centers.
L'archive ouverte pluridisciplinaire HAL, est destinée au dépôt et à la diffusion de documents scientifiques de niveau recherche, publiés ou non, émanant des établissements d'enseignement et de recherche français ou étrangers, des laboratoires publics ou privés. 


\title{
A New Cloud-Based $h p$ Finite Element Method
}

\author{
J. T. Oden*, C. A. M. Duarte ${ }^{\dagger}$ and O. C. Zienkiewicz ${ }^{\ddagger}$ \\ TICAM - Texas Institute for Computational and Applied Mathematics \\ The University of Texas at Austin \\ Taylor Hall 2.400 \\ Austin, Texas, 78712, U.S.A.
}

\begin{abstract}
A hybrid computational method for solving boundary-value problems is introduced which combines features of the meshless $h p$-cloud methods with features of conventional finite elements. The method admits straightforward nonuniform $h p$-type approximations, easy implementation of essential boundary conditions, is robust under severe distortions of the mesh, and can deliver exponential rates of convergence. Results of numerical experiments are presented.
\end{abstract}

\section{The Method}

Recent developments made in the context of meshless methods have demonstrated the simplicity of adding hierarchical refinements to a low order set of shape functions which satisfy the partition of unity (PU) requirement $[1-4,6,7]$. In particular, in the $h p$-cloud method introduced in [2], one covers the domain $\Omega$ of the solution of a boundary-value problem with a collection of open sets $\omega_{\alpha},\left(\bar{\Omega} \subset \cup_{\alpha=1}^{n} \omega_{\alpha}\right)$, the sets $\omega_{\alpha}$ being the clouds, and constructs on the clouds a set of global basis functions $\varphi_{\alpha}$ which form a PU on $\Omega$ :

$$
\sum_{\alpha=1}^{n} \varphi_{\alpha}(x)=1, \quad \boldsymbol{x} \in \bar{\Omega}
$$

\footnotetext{
*Professor, Director, TICAM. e-mail: oden@tıcam.utexas.edu

${ }^{\dagger}$ Research Assistant, TICAM. e-mail: armando@ticam.utexas.edu

$\ddagger$ Visiting Professor, TICAM; permanent address:University of Wales, Swansea
} 
One can then build spectral ( $p$-type) approximations by constructing products of the $\varphi_{\alpha}$ with higher-order spectral approximations to produce enriched basis functions $\varphi_{\alpha} L_{p}, L_{p}$ being a polynomial of degree $p$. This idea has been used successfully in generating exponentially convergent approximations of elliptic boundary-value problems in which convergence is obtained by appropriate $h$-refinement, $h=\max _{\alpha} h_{\alpha}, h_{\alpha}$ being the diameters of the clouds $\omega_{\alpha}$, or $p$-enrichment, $p$ being the order of the polynomial carried by the basis functions $\varphi_{\alpha} L_{p}[2-4,6,7]$. Here, the basic building block for such approximation is the PU, and, as observed in [1], this partition of unity can be furnished by a conventional finite element method. While such a PU on a finite element mesh will destroy the "meshless" quality of the approach, ample compensation for this loses is provided by a number of advantages over conventional $h p$-finite element methods.

Consider, for example, the conventional finite element meshes of triangles or quadrilaterals shown in Figs. 1 and 2, respectively, on which continuous global basis functions (shape functions) $N_{\alpha}$ are constructed at each nodal point $\boldsymbol{x}_{\alpha}, \alpha=$ $1,2, \ldots, n$. These functions are such that

$$
\sum_{\alpha=1}^{n} N_{\alpha}(\boldsymbol{x})=1, \quad \text { at any } \boldsymbol{x} \in \bar{\Omega}
$$

and thus form a PU. By setting $\varphi_{\alpha} \equiv N_{\alpha}$, we can build $h p$-cloud approximations on this PU and thereby produce a cloud basis that has all of the useful convergence properties of the $h p$-clouds but which combines features of conventional FEM's, such as exhibiting the Kronecker-delta property at boundary nodes. Some examples of cloud-type basis functions are shown in Figs. 3, 4 and 5. Notice from the figures that (see also Figs. 6, 7, 8)

- The clouds $\omega_{\alpha}$ need not be disks or even convex polygons,

- The mesh parameter $h$ (or $h_{\alpha}$ ) is the diameter of the cloud (the patch surrounding node $\boldsymbol{x}_{\alpha}$ ) and not the diameter of an element in the cloud

Figure 8 shows a conventional hierarchic field as used today in many codes and introduced in the manner described originally by Zienkiewicz, et al [11] and later elaborated on by others $[8,10]$.

(a) The conventional hierarchic polynomial are introduced using local, element based, co-ordinates. These provide complete Cartesian polynomials only up to the linear terms when isoparametric distortion is introduced. This completeness can be extended to quadrilateral elements using quadratic terms only by introduction of the 9 node expansion (as shown in [12]). For higher order terms, performance of elements may well deteriorate; viz [5]. 




Figure 1: Global finite element shape function $N_{\alpha}$ built on a mesh of triangles.

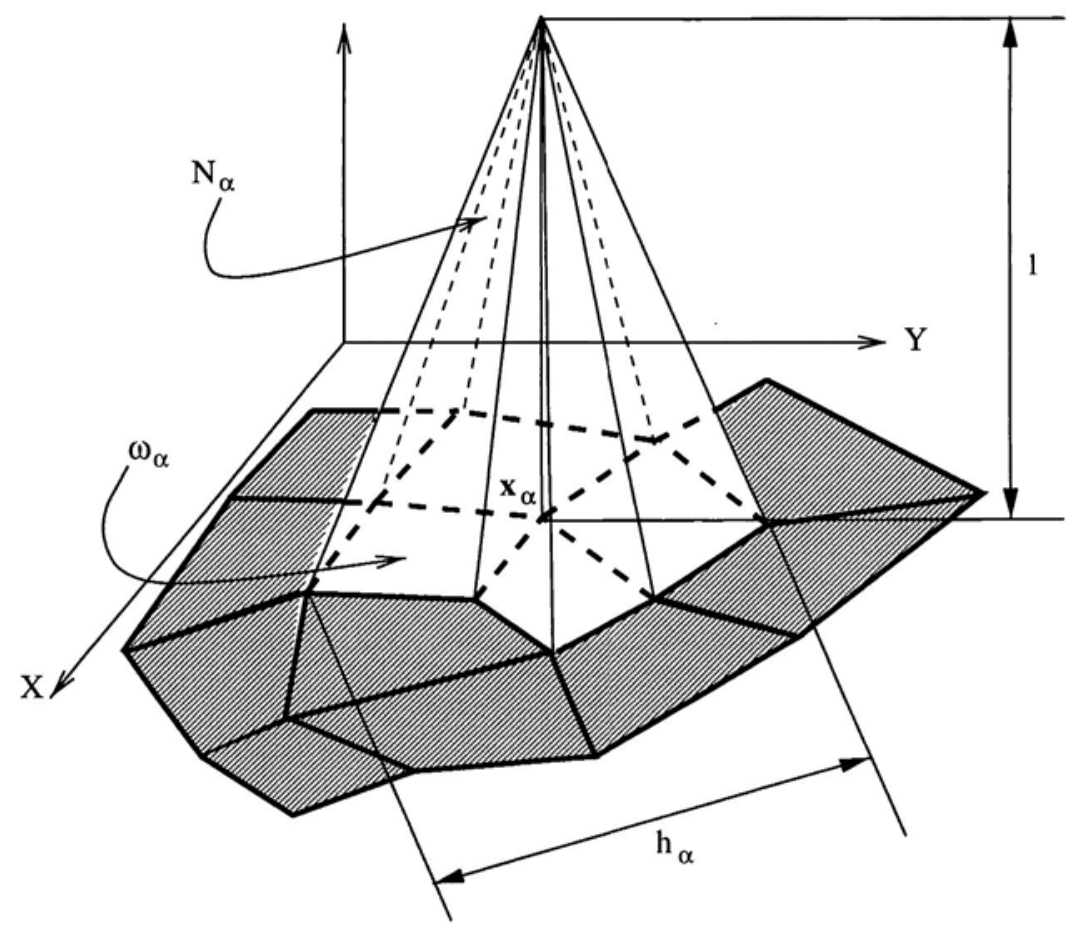

Figure 2: Global finite element shape function $N_{\alpha}$ built on a mesh of quadrilaterals. 




Figure 3: Bi-linear shape function associated with a node at $(0,0)$.

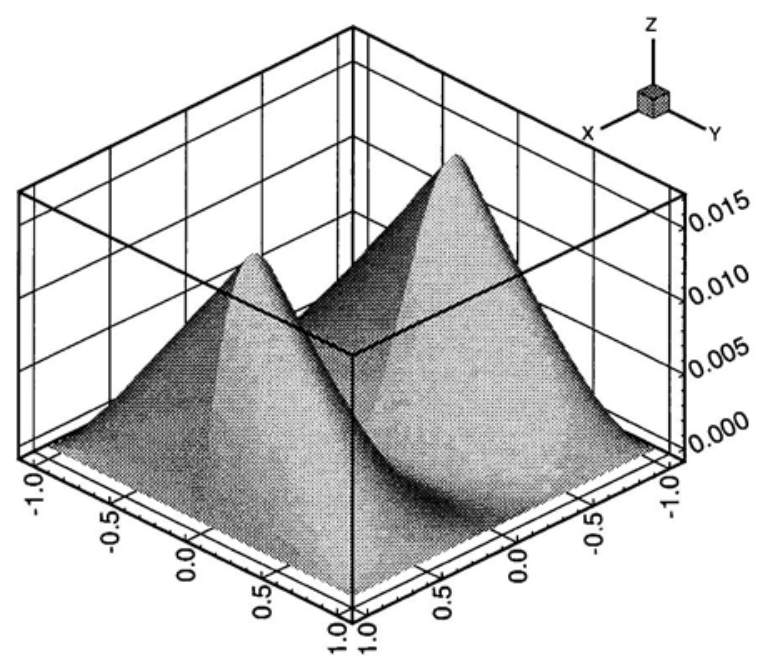

Figure 4: Higher order hierarchical shape function built from the product of the bilinear shape function shown in Fig. 3 and the monomial $x^{2}$. 


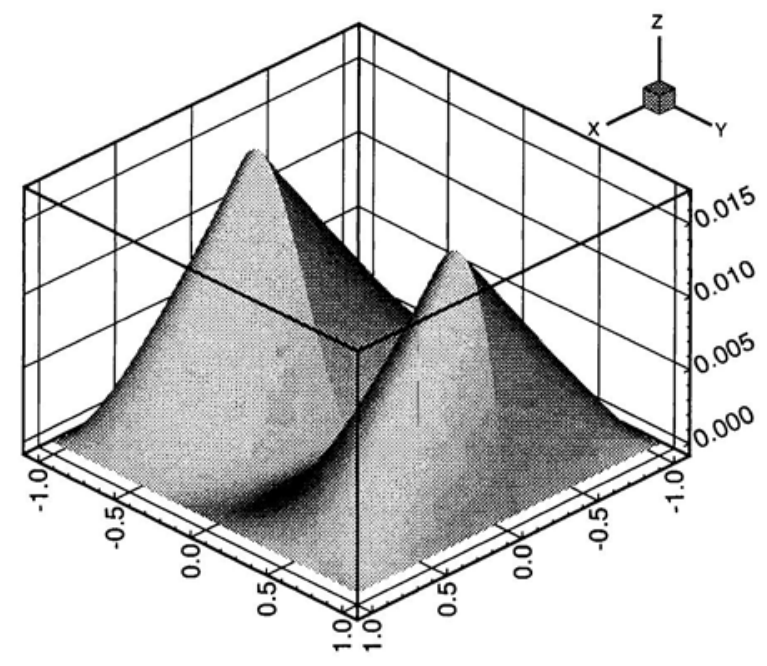

Figure 5: Higher order hierarchical shape function built from the product of the bilinear shape function shown in Fig. 3 and the monomial $y^{2}$.

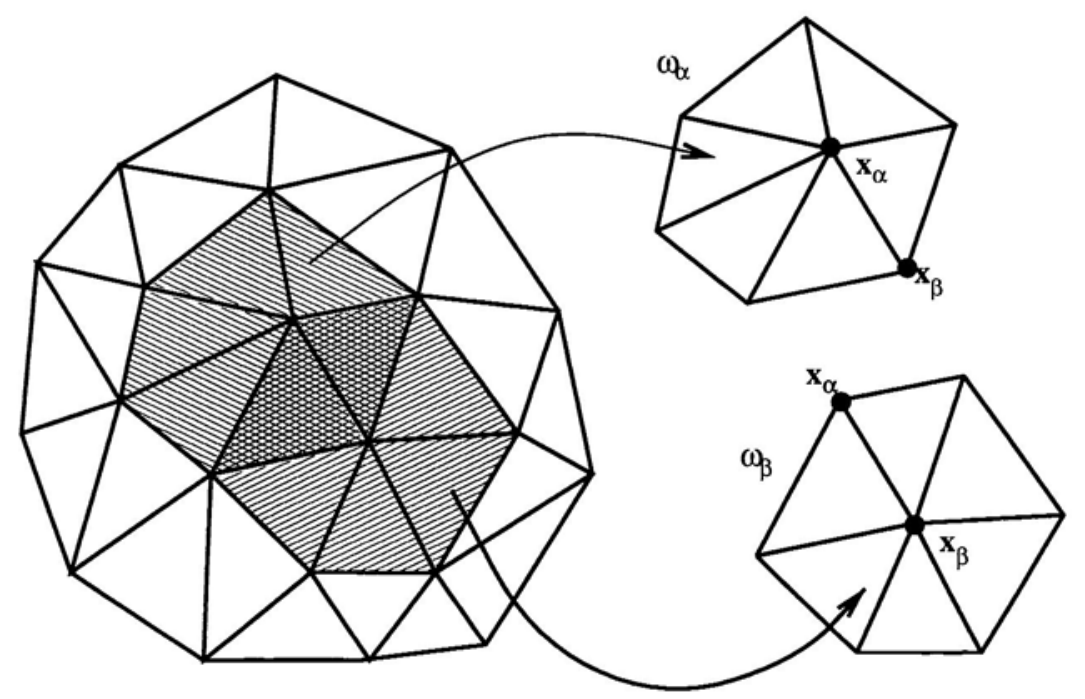

Figure 6: Overlapping patchs corresponding to clouds $\omega_{\alpha}$ and $\omega_{\beta}$. Polynomials of differing degree $p_{\alpha}$ and $p_{\beta}$ can be associated with nodes at $\boldsymbol{x}_{\alpha}$ and $\boldsymbol{x}_{\beta}$ so as to produce non-uniform $h p$-cloud/finite element approximations. 


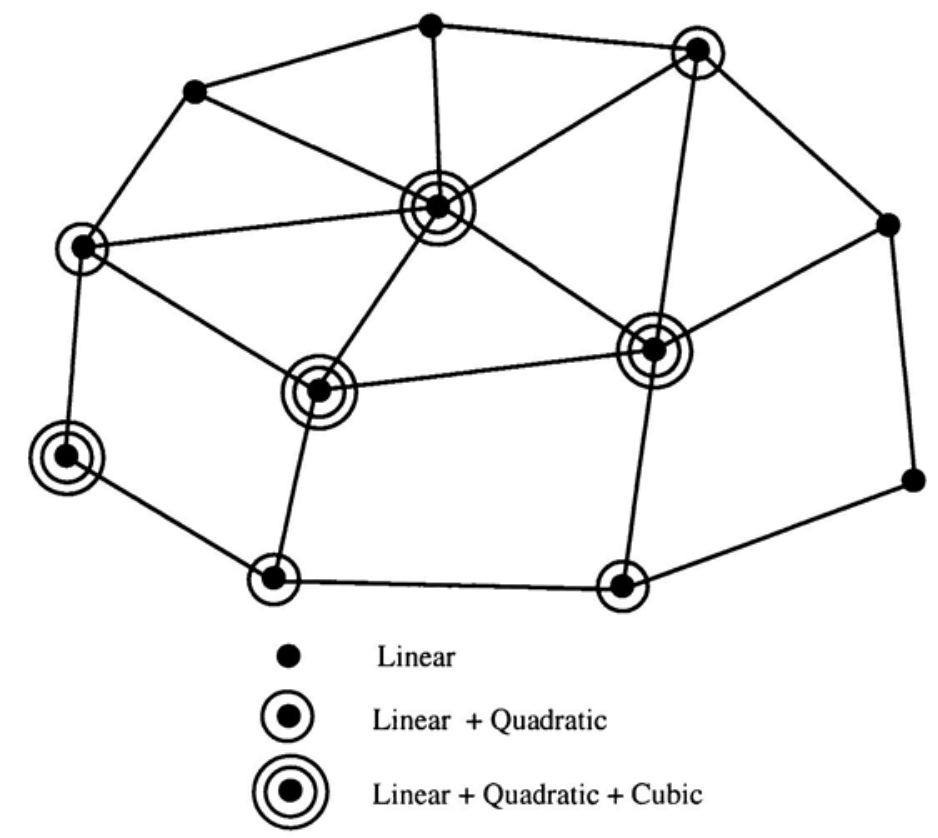

Figure 7: Cloud-based hierarchic fields in two dimensional space.

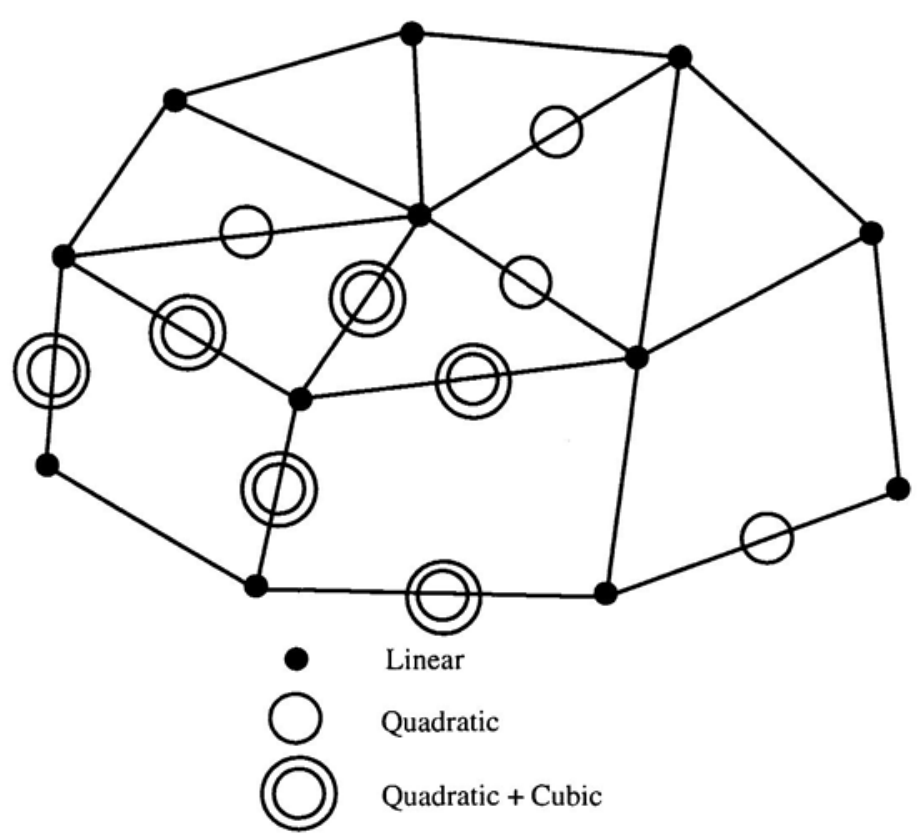

Figure 8: Conventional hierarchic fields in two dimensional space. 
On the contrary, the new cloud-based hierarchic forms give the terms of quadratic and higher order expansion in terms of Cartesian coordinates throughout and will always retain the accuracy corresponding to the spectral order. Indeed some saving in degrees of freedom necessary for a given accuracy is available.

(b) The new hierarchic form concentrates all the unknown degrees of freedom at corner nodes of the elements (cf. Fig. 7). This ensures a more compact band structure than that arising from the conventional hierarchic form.

(c) Remarkably, the structure of this approximation allows the use of different values of the spectral order $p$ on each cloud. Thus, other that basic connectivity of the low-order FEM mesh, none of the complications of high-order constraint conditions used in $h p$ - finite element methods are necessary. Non-uniform $h$ and $p$ approximation can be easily accommodated over the mesh, as suggested in Fig. 6 .

(d) Further, the elimination of corner degrees of freedom follows precisely the same pattern as that used for the underlying linear element repeating the same elimination steps in the equation solver. This means that vectorization/parallelization of the algorithms is relatively easy. What is more, if adaptive steps are used in the refinement, insertion of higher order terms at any node does not alter the computational sequence and can be simply accomplished.

(e) The new process presents no difficulties on the boundaries if Dirichlet (prescribed displacement) boundaries occur and no higher order terms are introduced in boundary nodes than a simple specification of corner displacement suffices.

With higher order terms on boundary nodes and with locally curved boundaries, additional constraints may be required (exactly as in standard hierarchic elements).

For Neumann (prescribed traction) boundaries, no such difficulties arise providing the integration is carried out on the exact curves.

\subsection{Construction of Linearly Independent Basis Functions}

The cloud-based $h p$ finite element basis functions are defined above as

$$
\phi_{\alpha i}:=N_{\alpha} L_{i}
$$

where $N_{\alpha}$ is a finite element shape function and $L_{i}$ is a polynomial of degree $p$. Since the shape functions form a $\mathrm{PU}$

$$
\sum_{\alpha} \phi_{\alpha i}=\sum_{\alpha} N_{\alpha} L_{i}=L_{i} \sum_{\alpha} N_{\alpha}=L_{i}
$$

Therefore, the polynomials $L_{i}$ can be recovered through linear combinations of the cloud basis functions $\phi_{\alpha i}$. The finite element shape functions have the property that 
exist $a_{x \alpha}, a_{y \alpha}, \in \mathbb{R}, \alpha=1, \ldots, n$ such that

$$
\begin{aligned}
& \sum_{\alpha} a_{x \alpha} N_{\alpha}=x \\
& \sum_{\alpha} a_{y \alpha} N_{\alpha}=y
\end{aligned}
$$

Now if we take $L_{i}=x$ or $L_{i}=y$ we have from (1) that

$$
\sum_{\alpha} N_{\alpha} x=x \quad \sum_{\alpha} N_{\alpha} y=y
$$

Therefore the set $\left\{N_{\alpha}, N_{\alpha} x\right\}_{\alpha=1}^{n}$ is not linearly independent. This can be avoided by a careful choice of the functions $L_{i}$ used to build the cloud basis functions. For example, the elements from the space $\operatorname{span}\left\{N_{\alpha}\right\}$ must not be used to build the cloud basis functions.

\section{Numerical Experiments}

\subsection{Illustration of the Basic Ideas}

As a first numerical experiment, we consider the solution of the following boundaryvalue problem

Find $u$ such that

$$
\begin{aligned}
-\Delta u & =0 \quad \text { in } \Omega=(-1,1) \times(-1,1) \\
-\frac{\partial u}{\partial n} & =-\frac{\partial}{\partial n} \operatorname{Re}\left(z^{2}\right) \quad \text { on } \partial \Omega
\end{aligned}
$$

where $x+i y=z \in \mathbb{C}$ and $\mathrm{Re}$ denotes the real part of. The value of $u$ is fixed at $(1,1)$ in order to make the solution unique.

Figure 9 depicts the solution $u$. The initial finite element discretization is represented in Fig. 10. It is composed of only two quadrilateral bi-linear finite elements. The corresponding finite element solution obtained with this discretization is depicted in Fig. 11 which has an error in the energy norm of

$$
\frac{\left\|u-u_{h p}\right\|_{E}}{\|u\|_{E}}=79.06 \%
$$

Quadratic shape functions like those depicted in Figs. 4 and 5 are then added to node 0 , as shown in Fig. 12. The corresponding finite element solution is shown in Fig. 13. 


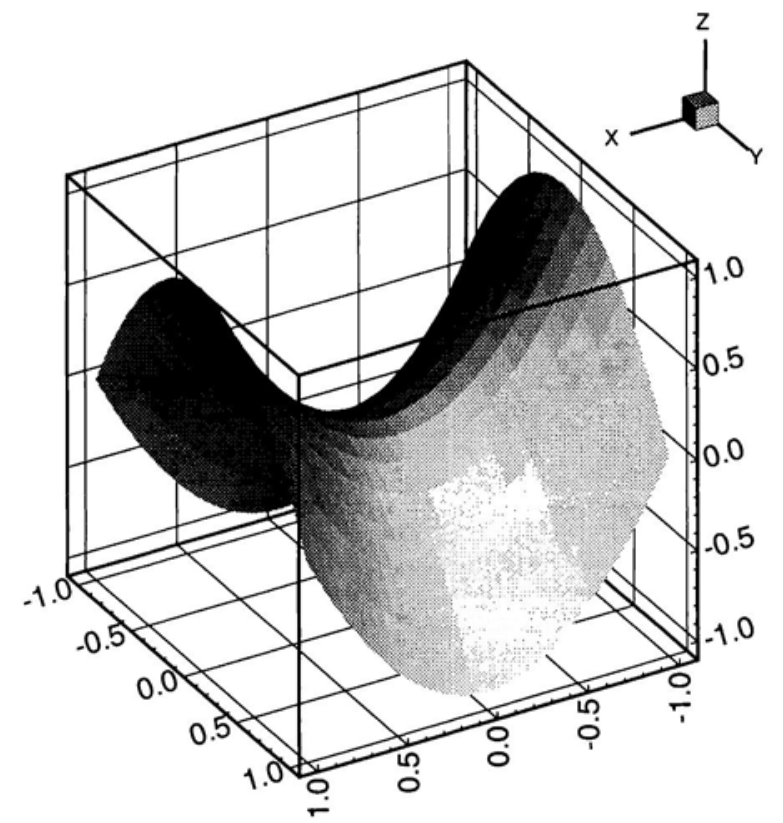

Figure 9: Solution of problem (2), (3).

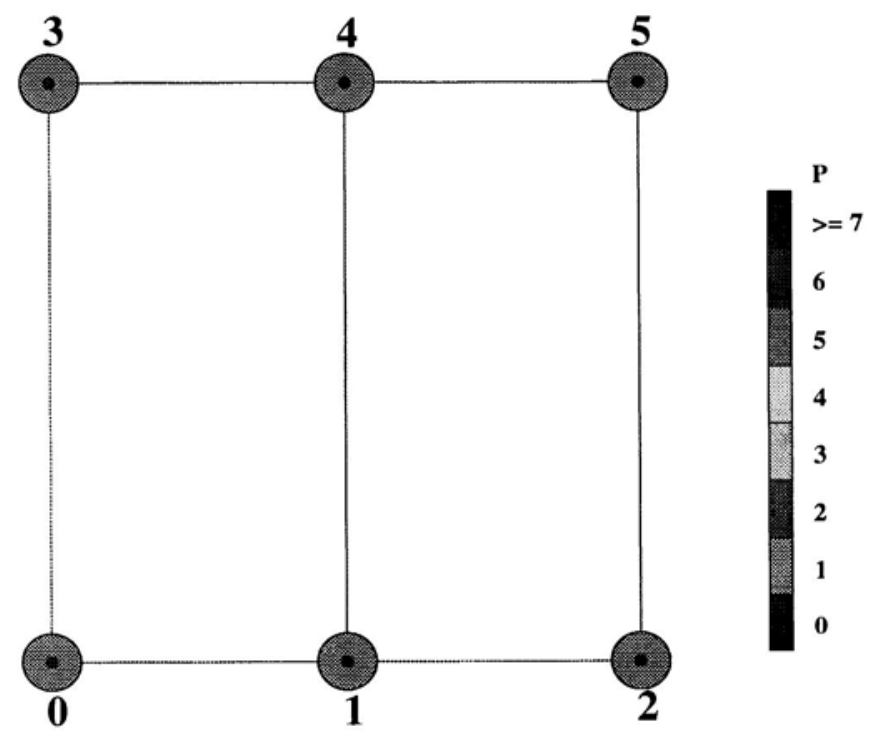

Figure 10: Initial finite element mesh composed of two bi-linear elements. 


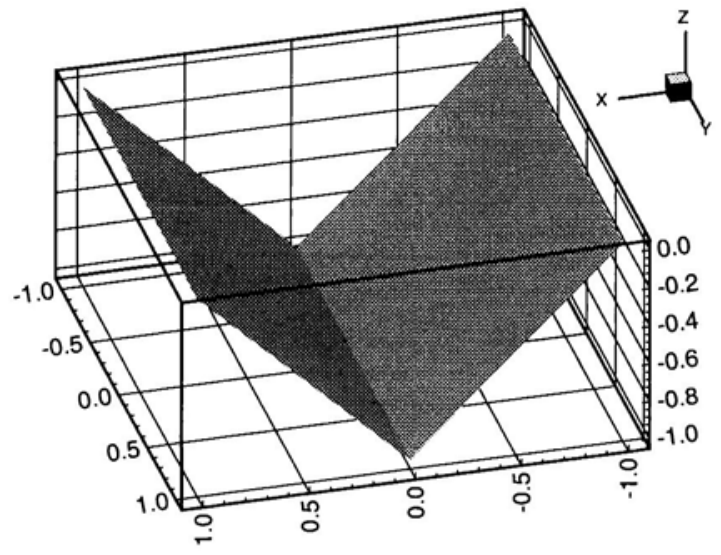

Figure 11: Finite element solution obtained using the discretization of Fig. 10.

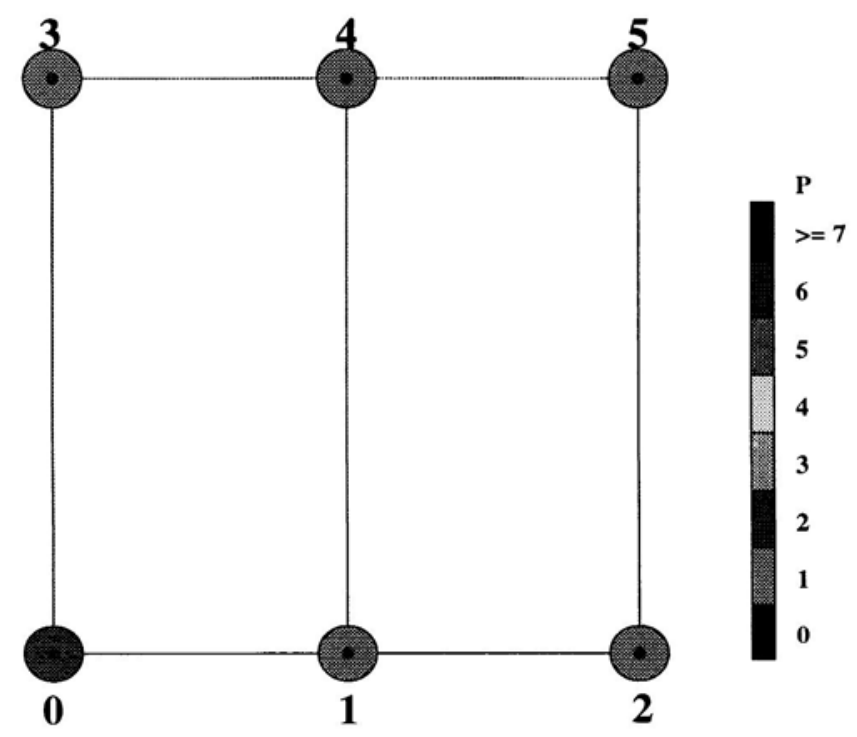

Figure 12: Cloud-finite element discretization with one quadratic node. 

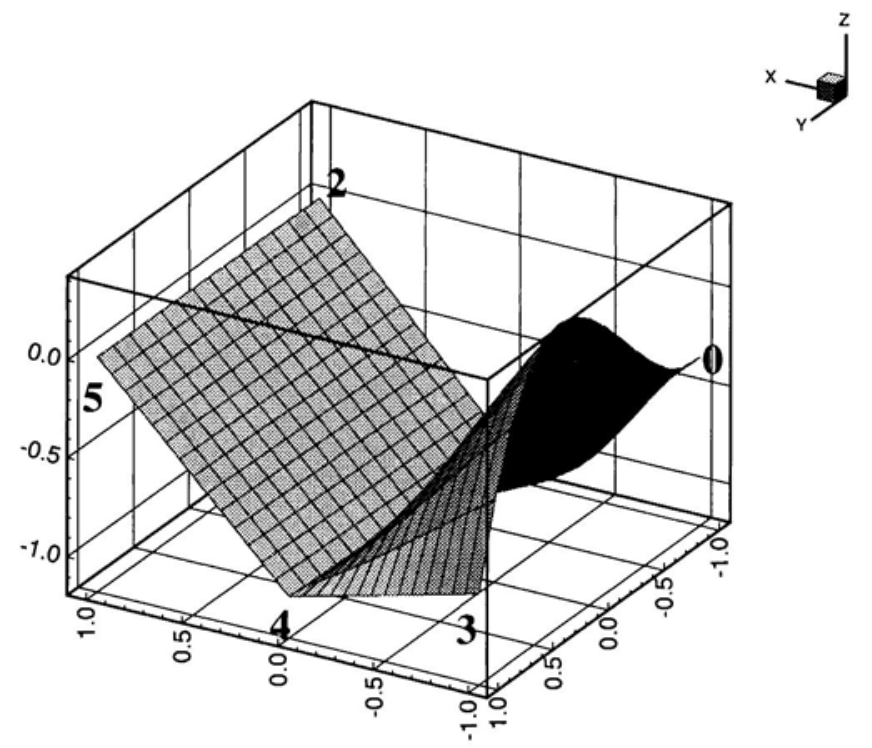

Figure 13: Finite element solution obtained using the discretization of Fig. 12.

Figure 14 shows the finite element discretization in which nodes $0,1,3$ and 4 have quadratic approximation associated with them, while nodes 2 and 5 have bilinear approximations. The corresponding finite element solution is shown in Fig. 15. The discretization error associated with this discretization is

$$
\frac{\left\|u-u_{h p}\right\|_{E}}{\|u\|_{E}}=34.78 \%
$$

Figure 16 shows the finite element solution obtained using quadratic shape function at all nodes of the discretization. As expected, the error for this discretization is of

$$
\frac{\left\|u-u_{h p}\right\|_{E}}{\|u\|_{E}}=\mathcal{O}\left(10^{-8}\right)
$$

It is emphasized that the enrichment of the finite element spaces, as described above, is done on a nodal basis and the polynomial order associated with a node does not depend on the polynomial order associated with neighboring nodes.

The insensibility of the cloud-based approximations to element distortion is next demonstrated. Figure 17 shows the finite element discretization used. All nodes have quadratic cloud-based approximations. Figure 18 shows the corresponding finite element solution. As before, we get

$$
\frac{\left\|u-u_{h p}\right\|_{E}}{\|u\|_{E}}=\mathcal{O}\left(10^{-8}\right)
$$




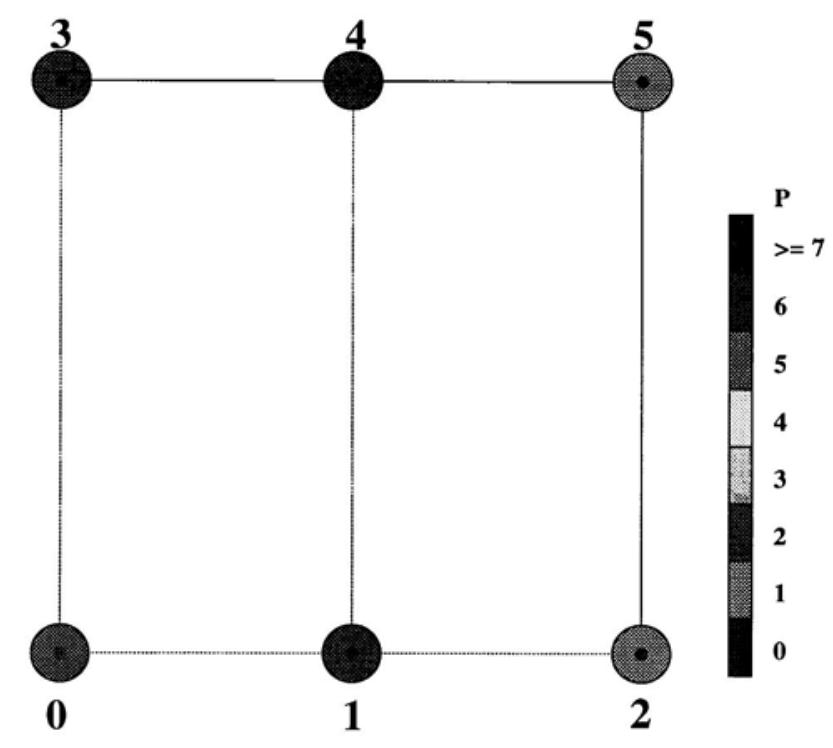

Figure 14: Cloud-finite element discretization with four quadratic nodes.

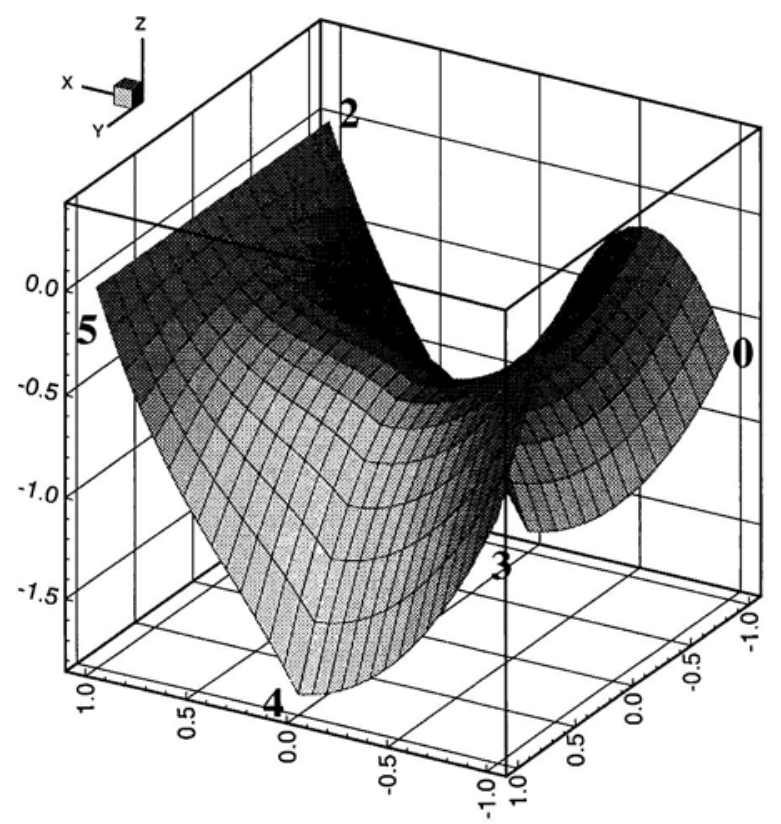

Figure 15: Finite element solution obtained using the discretization of Fig. 14. 


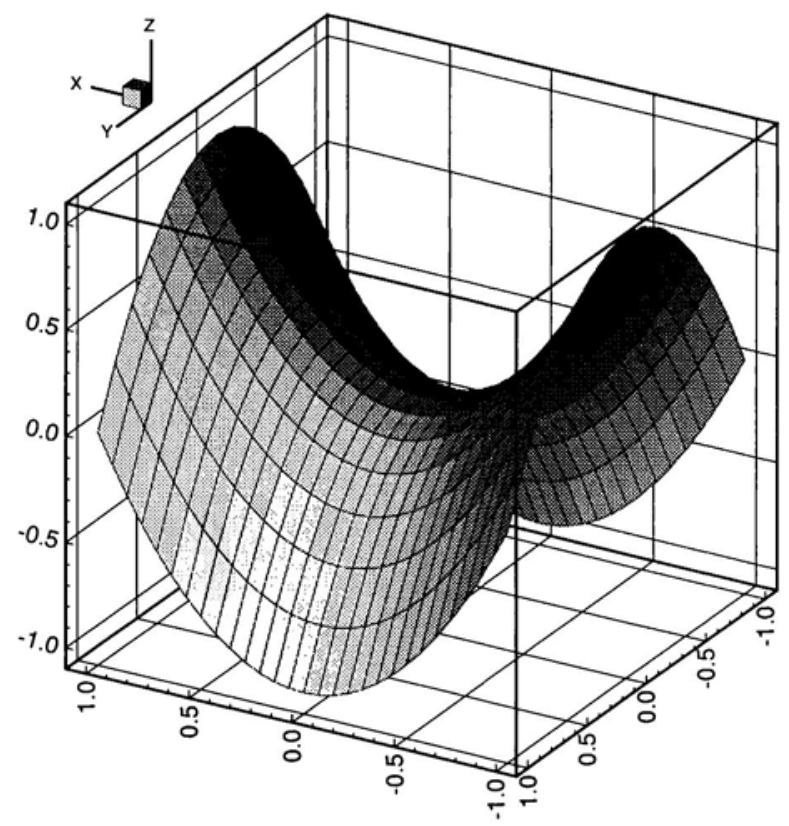

Figure 16: Finite element solution obtained using quadratic shape functions at all nodes of the mesh.



Figure 17: Distorted mesh of quadratic cloud-finite elements. 


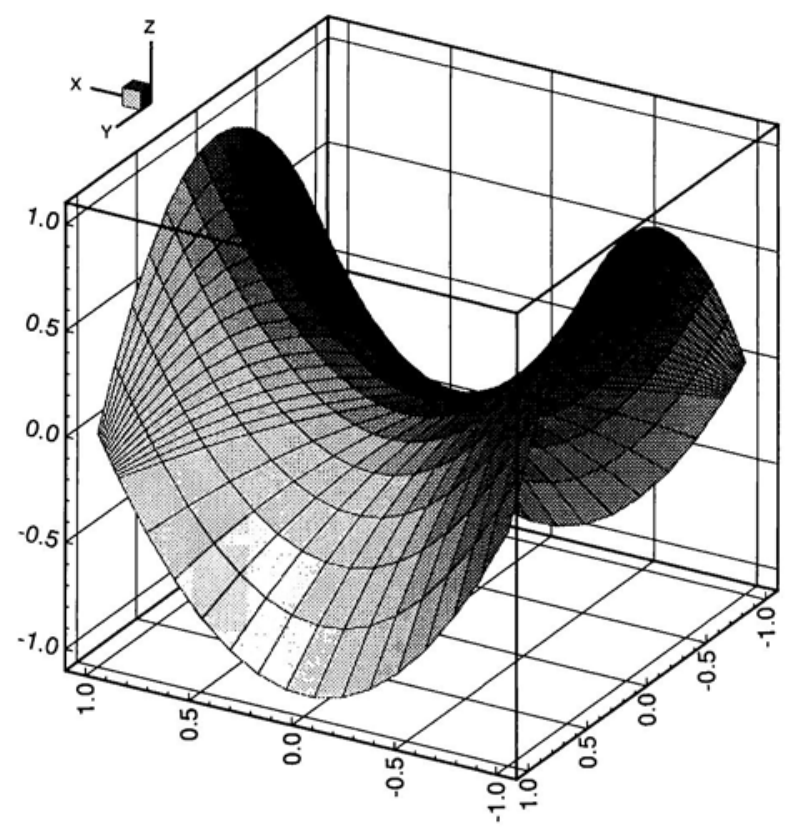

Figure 18: Finite element solution obtained using the discretization of Fig. 17.

\section{$2.2 \quad h p$ adaptivity}

We shall now consider the problem of an L-shaped plane elastic body loaded by the tractions associated with the following stress field

$$
\begin{aligned}
& \sigma_{x x}(r, \theta)=\lambda r^{\lambda-1}[(2-Q(\lambda+1)) \cos (\lambda-1) \theta-(\lambda-1) \cos (\lambda-3) \theta] \\
& \sigma_{y y}(r, \theta)=\lambda r^{\lambda-1}[(2+Q(\lambda+1)) \cos (\lambda-1) \theta+(\lambda-1) \cos (\lambda-3) \theta] \\
& \sigma_{x y}(r, \theta)=\lambda r^{\lambda-1}[(\lambda-1) \sin (\lambda-3) \theta+Q(\lambda+1) \sin (\lambda-1) \theta]
\end{aligned}
$$

where $(r, \theta)$ is the polar coordinate system shown in Fig. $19, \lambda=0.544483737$, $Q=0.543075579$.

The stress field (4) corresponds to the first term of the symmetric part of the expansion of the elasticity solution in the neighborhood of the corner $A$ shown in Fig. $19[9]$.

Plane strain conditions, unity thickness and Poisson's ratio of 0.3 are assumed. The strain energy of the exact solution is given by [9]

$$
\mathcal{E}(u)=4.15454423 \frac{a^{2 \lambda}}{E}
$$

where $E$ is the modulus of elasticity and $a$ is the dimension shown in Fig. 19. The values $E=a=1$ are assumed in the calculations. 




Figure 19: L-shaped elastic body.

Two sequences of discretizations, $\mathcal{S}_{1}$ and $\mathcal{S}_{2}$, are used to solve this problem. In the former, the uniform mesh shown in Fig. 20 is used and the polynomial order of the approximations ranges from 1 to 8. A strongly graded mesh, as shown in Fig. 21, and non-uniform $p$ distributions are used in the second sequence of discretizations. Geometric factors $q=0.10$ and $q=0.15$ for the size of the elements are used (cf. Fig. 21 ). Figures 22 and 23 show the $p$ distribution used in the fourth step of this sequence. The polynomial order of the clouds decrease linearly towards the singularity while the size of the finite elements decrease geometrically.

The relative error, measured in the energy norm, versus the number of degrees of freedom is shown in Fig. 24 for the sequences of discretizations $\mathcal{S}_{1}$ and $\mathcal{S}_{2}$ (with $q=0.10$ and $q=0.15$ ). As expected, the uniform mesh gives an algebraic rate of convergence while the strongly graded meshes lead to an exponential rate of convergence. This kind of behavior is typical of $h p$ - finite element methods. However, the construction of non-uniform $h$ - and $p$-discretizations in a cloud based framework is considerably more straightforward than in conventional $h p$ - finite element methods. For this problem, the strongly graded mesh with $q=0.10$ gives slightly better results than the case $q=0.15$ 


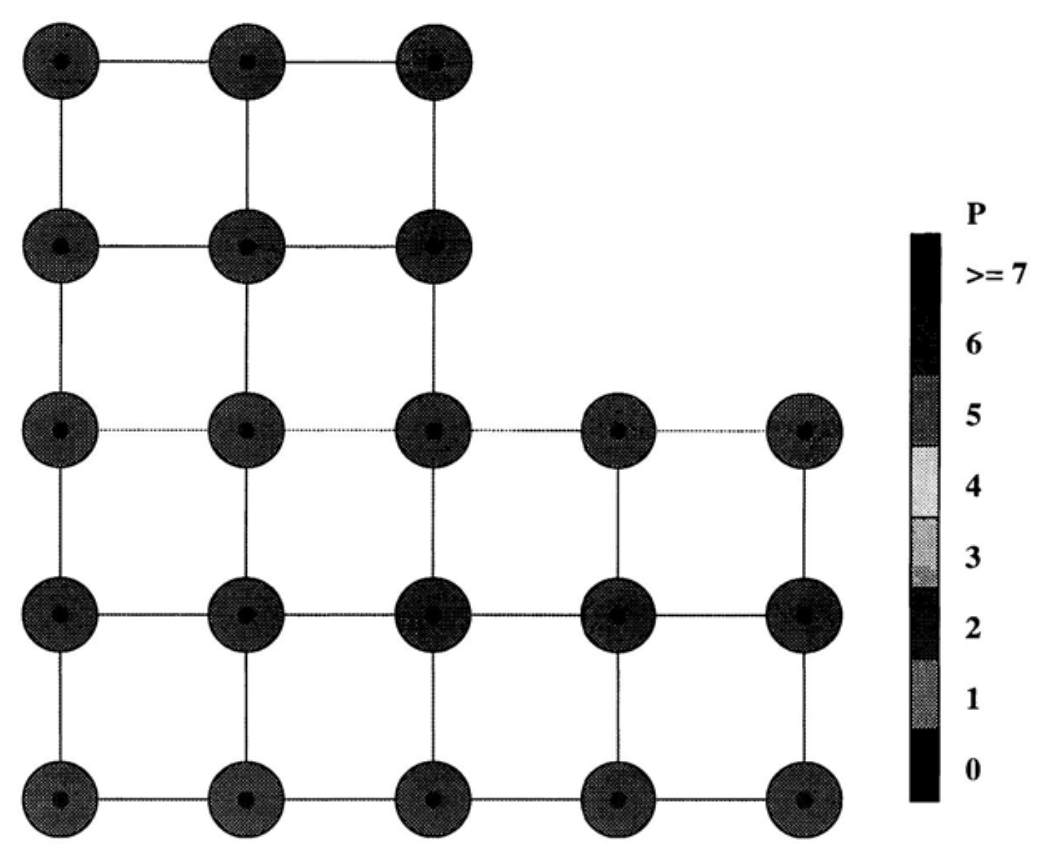

Figure 20: Uniform mesh and $p$ distribution for the L-shaped body.

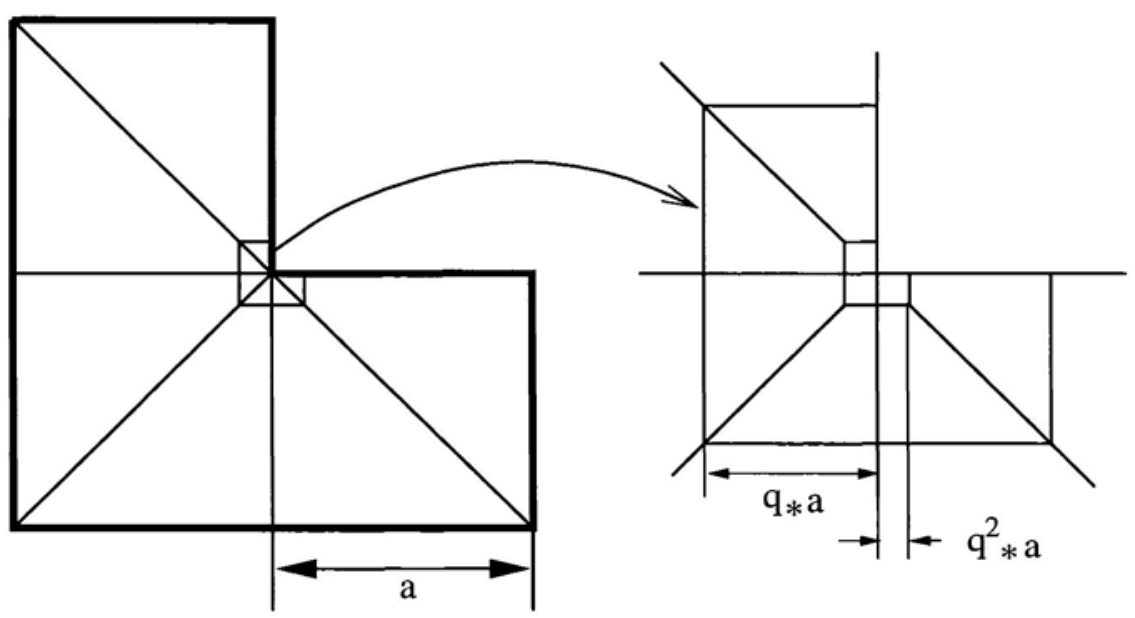

Figure 21: Geometric mesh for the L-shaped body. A geometric factor of $q=0.15$ is adopted in the computations. 


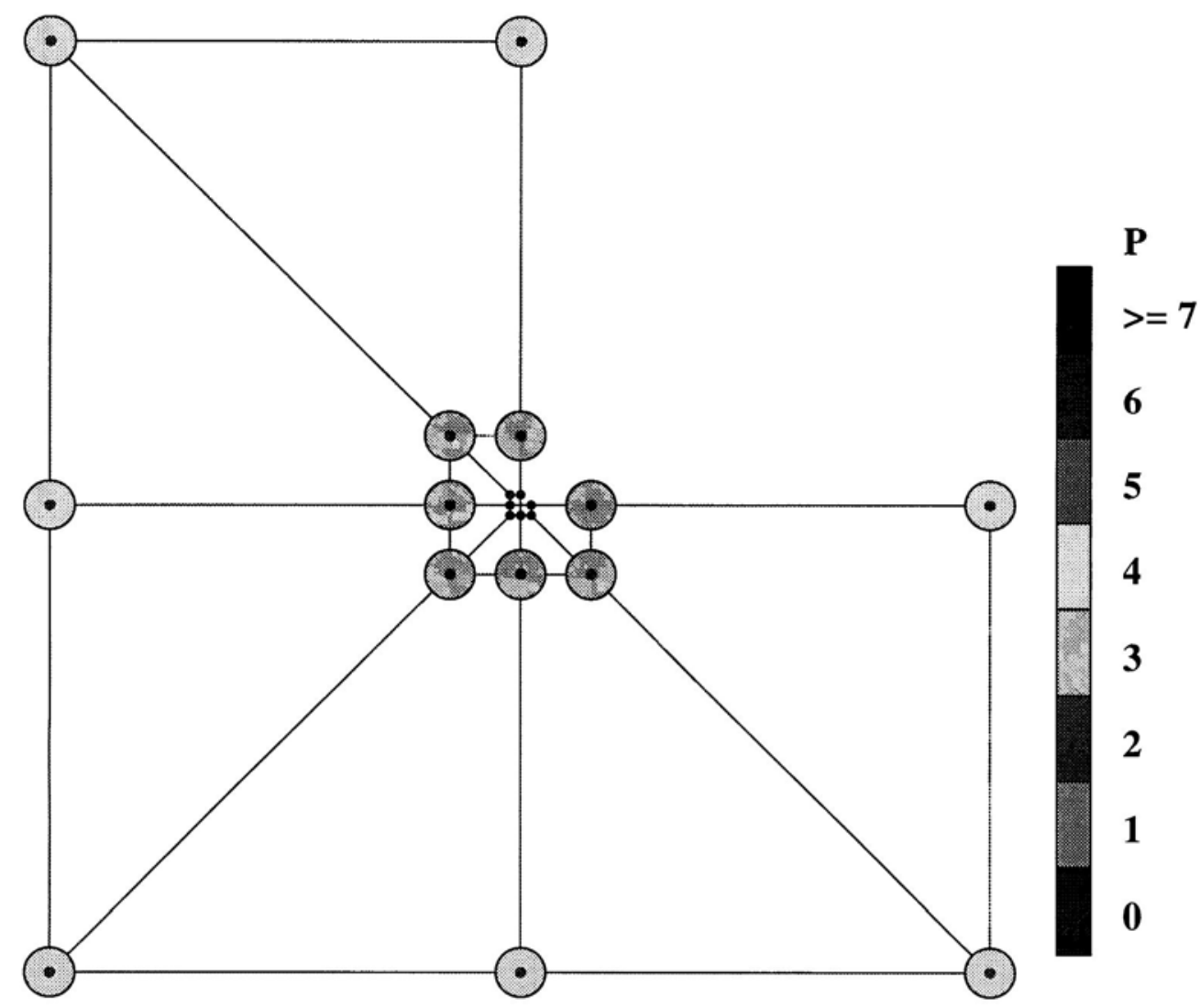

Figure 22: Polynomial order associated with the clouds at the fourth step of the sequence of discretizations $\mathcal{S}_{2}$.

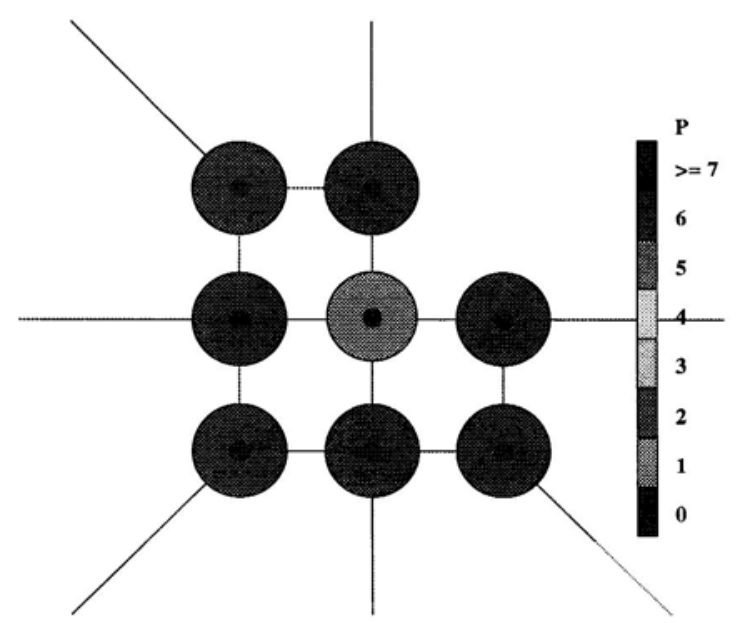

Figure 23: Zoom at clouds near the re-entrant corner of Fig. 22. 


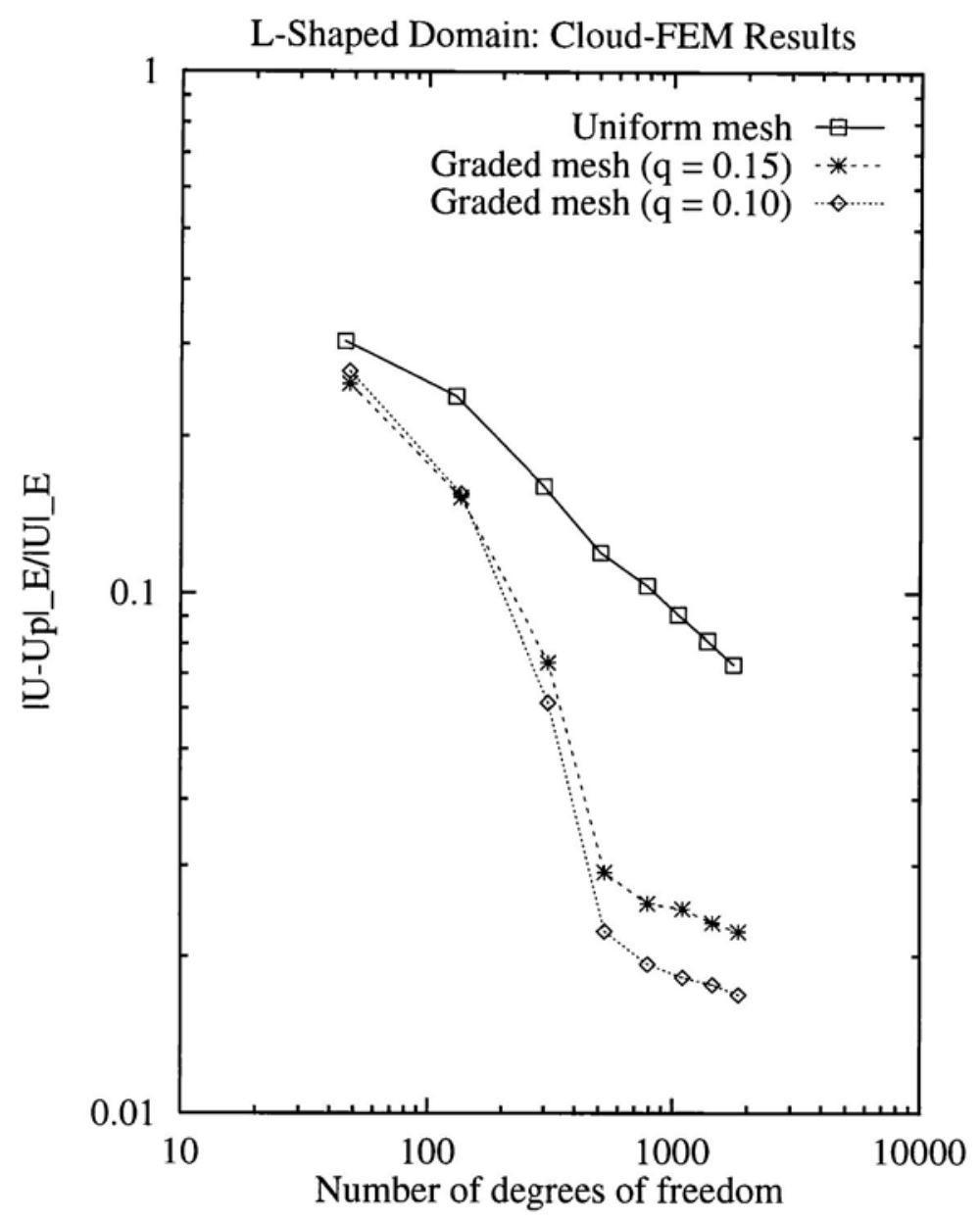

Figure 24: Convergence in the energy norm for uniform and non-uniform cloud-based $h p$ - distributions. 


\section{Conclusions}

In this investigation, we have explored the use of lower-order finite element approximations to generate partitions of unity on which hierarchical $h p$ - cloud approximations can be constructed. The resulting methodology has a number of useful features. Among these are that non-uniform $h p$ - meshing with variable and hierarchical order $p$ over clouds can be easily generated. The spectral convergence of $p$ - and $h p$-methods is retained and the method is very robust, the accuracy being quite insensitive to mesh distortion. Also, by building cloud approximations on finite element meshes, Dirichlet boundary conditions are easily handled.

The hp- convergence properties seem to differ from traditional $p$ - version elements, but exponential convergence is attained. Applications to problems with singularities are easily handled using cloud schemes. In all, this hybrid finite-element/cloud methodology appears to have a number of useful and attractive features that could prove to be important in broad engineering applications.

Acknowledgment: The support of this work by the the Army Research Office (J. T. Oden and C. A. Duarte) under contract DAAH04-96-0062 and the CNPq Graduate Fellowship Program (C. A. Duarte) grant \# 200498/92-4 are gratefully acknowledged.

\section{References}

[1] I. Babuska and J. M. Melenk. The partition of unity finite element method. Technical Report BN-1185, Inst. for Phys. Sc. and Tech., University of Maryland, June 1995.

[2] C. A. M. Duarte and J. T. Oden. Hp clouds-a meshless method to solve boundary-value problems. Technical Report 95-05, TICAM, The University of Texas at Austin, 1995.

[3] C. A. M. Duarte and J. T. Oden. An $h p$ adaptive method using clouds. Technical report, TICAM, The University of Texas at Austin, 1996. To appear in Computer Methods in Applied Mechanics and Engineering.

[4] C. A. M. Duarte and J. T. Oden. Hp clouds - an $h p$ meshless method. Numerical Methods for Partial Differential Equations, 12:673-705, 1996.

[5] Nam-Sua Lee and Klaus-Jurgen Bathe. Effects of element distortions on the performance of isoparametric elements. International Journal for Numerical Methods in Engineering, 36:3553-3576, 1993. 
[6] J. M. Melenk and Ivo Babuska. The partition of unity finite element method: Basic theory and applications. Computer Methods in Applied Mechanics and Engineering (to appear).

[7] J. T. Oden and C. A. M. Duarte. Solution of singular problems using $h-p$ clouds. In MAFELAP 96, 1996.

[8] A. G. Peano. Hierarchics of conforming finite elements for elasticity and plate bending. Comp. Math. and Applications, 2, 1976.

[9] B. A. Szabo and I. Babuska. Computation of the amplitude of stress singular terms for cracks and reentrant corners. In T. A. Cruse, editor, Fracture Mechanics: Nineteenth Symposium, ASTM STP 969, pages 101-124, 1988.

[10] O. C. Zienkiewicz, J. P. de S. R. Gago, and D. W. Kelly. The hierarchical concept in finite element analysis. Computers and Structures, 16:53-65, 1983.

[11] O. C. Zienkiewicz, B. M. Irons, J. Campbell, and F. C. Scott. Three dimensional stress analysis. In High Speed Computing in Elasticity, IUTAM Symp., pages 413-432, Univ. of Liege, 1970.

[12] O. C. Zienkiewicz and R. L. Taylor. The Finite Element Mehtod, $4^{\text {th }}$ Edition, volume I. McGraw-Hill, 1981. 